http://jmscr.igmpublication.org/home/ ISSN (e)-2347-176x ISSN (p) 2455-0450 crossref DOI: https://dx.doi.org/10.18535/jmscr/v7i8.10

Journal Of Medical Science And Clinical Research

\title{
Quality of life in post stroke patients with early rehabilitation intervention:
}

\section{A cross sectional study}

Authors

\section{Dr PC Muralidharan", Dr Vipin Kumar K ${ }^{2}$ Dr G Chitra ${ }^{3}$, Dr K Sreejith ${ }^{4}$, Dr S Abdul Gafoor ${ }^{5}$}

${ }^{1}$ Asst Professor, Dept of Physical Medicine and Rehabilitation. Govt Medical College, Kottayam

${ }^{2}$ Junior Resident, Govt Medical College, Kottayam

${ }^{3}$ Asst Professor, Govt Medical College, Kottayam

${ }^{4}$ Associate Professor, Govt Medical College, Kottayam

${ }^{5}$ Professor \& Head Dept of Physical Medicine and Rehabilitation, Govt Medical College, Kottayam

\begin{abstract}
Background: Rehabilitation enhances neurological recovery in stroke and improves the functional status. It is vital in the treatment of stroke. The quality of life depends on various physical, mental and psycho social factors and functional independence. Quality of life in a stroke survivor is a measure of success of rehabilitation. There are very few studies associating initiation of rehabilitation and quality of life in post stroke patients.
\end{abstract}

Objectives: The study aims to assess the difference in quality of life in stroke subjects who received early rehabilitation and in those who did not. This study also evaluates the association of demographic and physical variables with early rehabilitation in a stroke survivor.

Materials and Methods: A cross sectional study conducted in the outpatient clinic of Department of Physical Medicine and Rehabilitation, Government Medical College Kottayam among the post stroke patients 3-6 months after the first episode of stroke. Data was collected from the patients through questionnaire to assess the socio-demographic variables and stroke specific quality of life (SSQOL).

Results: Of the 200 subjects recruited for the study, 141 received early rehabilitation and their mean total SSQOL score was 182.82 while the mean score for the group which did not receive early rehabilitation was 159.29 , which was statistically significant $(p<0.05)$. The mean age in the group who received early rehabilitation was less than the other, which was statistically significant. The other demographic variables did not show significant association with early rehabilitation. Pain and independence in gait showed significant association with early rehabilitation.

Conclusion: Subjects who received early rehabilitation after stroke had better quality of life than those who did not. Moreover those who had early rehabilitation were more independent in gait and had better perception of pain compared to others. The study calls for the initiation of rehabilitation early in the stroke treatment pathway.

Keywords: Stroke, Early rehabilitation, Quality of life.

\section{Introduction}

Stroke is a sudden occurrence of a non-traumatic brain injury caused by occlusion or rupture of cerebral blood vessels leading to focal neurological deficits characterised by loss of motor control, altered sensations, cognitive or 
language impairment, disequilibrium or coma. The sudden onset of stroke causes a dramatic change in the various domains of life in the individual ${ }^{1}$. Of the 15 million people affected with stroke worldwide, 5 million lives with permanent disabilities of different degrees ${ }^{2}$. India reports a yearly incidence of 165000 stroke $^{3}$. The annual incidence rates of stroke observed in India exceeds the US and Europe ${ }^{4}$. Stroke related mortality and morbidity persists as a significant public health issue in all developing countries. A well organised specialist care starting from advanced acute care through to structured rehabilitation program constitutes the treatment pathway for stroke. Stroke affects the lifestyles of the survivor leading to a decline in the functional status and worsens their quality of life ${ }^{1}$. It is the third leading cause of disability ${ }^{5}$, and its sequel increases the burden on the survivor, family, care giver and the society at large.

With the advent of rapid advancements in acute and rehabilitation treatment facilities in stroke, the physical and functional status of the stroke survivor has shown significant improvements. But they have to cope up with different grades of physical, psychological, social and functional sequel which influences their quality of life.

Rehabilitation forms the cornerstone of treatment programs in multidisciplinary stroke care ${ }^{6}$, limiting the development of disabilities. This facilitates the stroke survivor to attain an optimal functional ability, provides functional independence and improvement in quality of life. This also provides an improved interaction with the family, society and the environment. The improved outcomes are influenced by timing, duration and quality of rehabilitation services available to the patients ${ }^{7}$. Strokes occur more frequently in the low and middle income countries like India where the scope for structured rehabilitation is limited due to technical and economic reasons, unlike the West ${ }^{8}$.

Rehabilitation is a relearning process which enhances and augments natural recovery mechanisms. This involves oedema resolution, vicariation of function and reversal of diaschisis 9 About $70 \%$ of recovery sets in the first three months ${ }^{9}$. This phase is a period of brisk spontaneous recovery and the repair processes of synaptogenesis, angiogenesis and neurogenesis are intense. A minimum of spontaneous activity and therapy facilitates proportional recovery to occur except in corticospinal tracts which is late to recover. For a greater extent of recovery, higher intense therapy is essential. Skill learning, active participation and motor retraining and environment enrichment- all increases neuronal plasticity, network activation and subsequent functional recovery. The duration of maximal neuronal plasticity is not well defined ${ }^{10}$ and the rehabilitation process is construed as continuous.

Early neurological rehabilitation is an important phase of stroke rehabilitation. This is found to accelerate recovery, minimise complications, have positive psychological influences and provide effective support system for the stroke survivor. The critical period for initiation of rehabilitation is not defined, though the first thirty days have been considered significant ${ }^{11}$. Early rehabilitation is thought of as a part of total care plan. The central nervous system responds to injury by structural and functional recombination and neuronal plasticity during this early phase. Studies have established correlation between early rehabilitation and functional recovery as well as with improved quality of life. Though there is no definite agreement on the time of commencement of rehabilitation, studies have been undertaken to observe the results of early and very early rehabilitation varying from less than 24 hours to one month . Initiation of rehabilitation at various points of time i.e. <24 hours, $24-48$ hours, within 72 hours, 2 weeks and 30 days were subjects of study to evaluate the outcome ${ }^{10,12}$. Rehabilitation initiated within the first 2 weeks have reported beneficial effects ${ }^{10}$. Though very early mobilisation, $<24$ hours, was considered to have beneficial effects in stroke, studies reported harmful effects ${ }^{10}$. Commencement of rehabilitation as soon as the patient becomes 
medically stable helps to augment functional gains and provides the advantage of the period of early recovery ${ }^{8}$. Kottke.Is highlighted the role of early intervention in improving the quality of life in post stroke patients ${ }^{13}$.

Quality of life is multidimensional and is a vitally important outcome for stroke survivors influencing their physical, mental and psychosocial functions. WHO defines quality of life as an individual's perception of their position in life relative to the culture and values and in relation to their standards, goals, expectations and concerns $^{14}$. This is an analysis of a person's physical and emotional functions, memory, social and personal interactions including locomotion and communication and thought capacities ${ }^{15}$. It assumes significance in the community health care settings ${ }^{16}$ and is also a measure of successful rehabilitation ${ }^{17}$.

Quality of Life (QOL) is influenced by factors like age, gender, severity of lesion, functional deficits, quality of rehabilitation etc. It can be assessed by means of general and specific scales. SF36, Euro QOL 5 etc. are general scales while SSQOL and Stroke impact scales are specific ones for stroke.

SSQOL is a patient centered assessment scale devised by Williams et. $\mathrm{al}^{18}$ and many versions of this is validated in many languages. This envisages quality of life in stroke survivors in different functional spheres - physical, psychological, functional and social. The quality of life in the stroke survivor is evaluated in patient's perspective and gives an insight into the needs and demands of that individual with respect to his physical and mental state, social factors, independence in activities of daily living and interactions. Many studies have reported decline in the QOL after stroke and this was based on the degree of independence in activities of daily living and social and personal participation. Most of the stroke survivors have sequel involving motor, sensory, cognition, speech, equilibrium or balance. These show variable responses to treatment. The outcome is determined by severity and extent of lesions, presence of comorbidities and risk factors, acute care facilities, complications, cognitive status, family and care giver support and proper rehabilitation. Several factors like socio economic status and educational status influences the rehabilitation outcome.

The quality of life is a reflection of the success of the rehabilitation process prevalent in the community. The outcome in stroke after rehabilitation indicates the appropriateness of different treatment strategies involving the various domains affected in stroke. SSQOL throws light on this aspect in the perspective of the patient which also should be taken into consideration while deciding the treatment plan. In view of the very limited number of studies available assessing the QOL and the effect of early rehabilitation, this study was conducted.

The primary objective of the study was to assess the difference in quality of life among the stroke survivors who received early rehabilitation and those who did not. This also attempted to find out the variations in the various domains of the quality of life between these groups. The secondary objective of the study was to find out the association of demographic and physical variables with subjects who underwent early rehabilitation and those who did not.

\section{Methodology}

Study design was cross sectional study. Study was done at outpatient clinic, Department of Physical Medicine \& Rehabilitation, Government Medical College, Kottayam. The patients who reported to the clinic from October 2018 to March 2019 (6months) were included in the study. Diagnosis of stroke was confirmed by clinical and/or radiological findings and perusal of the available records. The study was approved by the institutional ethical review board and informed consent was taken from all subjects. Sample size was calculated as 200 and it was calculated by using following formula: $\mathrm{n}=\left(\mathrm{Z}^{2} \times \mathrm{P}(1-\mathrm{P})\right) / \mathrm{e}^{2}$ where $\mathrm{Z}=$ value from standard normal distribution corresponding to desired confidence 
level $(\mathrm{Z}=1.96$ for $95 \% \mathrm{CI}), \mathrm{P}$ is expected true proportion kept as $49.6 \%$ the level of precision at $15 \%$. Subjects were included if they had stroke, with a duration 3-6 months after the first episode of stroke. Subjects with concomitant neurological diseases, traumatic brain injury and tumor, severe cardiac, hepatic or renal diseases were excluded. Those patients with impaired comprehension and aphasia and with recurrent stroke were also excluded from the study. The subjects were excluded if they were unwilling to cooperate. The data was collected using the Proforma to include demographic, physical variables and outcome variable such as early rehabilitation (yes/no) and the translated version of SSQOL scale in Malayalam. Demographic data included were age, gender, education, side and type of lesion and physical variables included were speech and pain impairment, treatment for depression and gait. Those who did not receive early rehabilitation were coded as 0 and those who received were coded as 1. A comparative evaluation was performed between subjects who did not receive early rehabilitation belonging to group $\mathrm{A}$ and subjects who received early rehabilitation belonging to group B and data was analysed.

Outcome measure: SSQOL is a self-reported questionnaire specific for stroke, translated and validated in many countries. It has 49 items in 12 domains with three response sets in 5 point Likert format. This covers the domains of energy, family roles, language, mobility, mood, personality, selfcare, social roles, thinking, upper extremity function, vision and work and productivity. The different responses are based on the amount of help required (no help to total help), amount of trouble experienced (unable to do to no trouble) and the degree of agreement with statements regarding functions (strongly agree to strongly disagree).This covers each of the domains mentioned. The minimum score is 49 and the maximum is 245 . The higher the score, better will be the quality of life. A score of less than $60 \%$ (147) is defined as low quality of life $^{19}$. Independent predictors of good overall HRQOL
(Health Related Quality of Life) were the SSQOL score Odds Ratio 2.97; 95\% CI, 1.3, 7.1; p= 0.0122 .

Data Analysis: Data analysis was performed by SPSS (version 17) for windows. Unpaired t test was used to find out whether the age has influenced quality of life in stroke patients between groups (group A: who didn't receive early rehabilitation, group B who received early rehabilitation). Chi-square test was used to find out the association of demographic variables such as gender, side, site, type of lesion, education, occupation and physical variable such as speech, pain, depression and gait (independent or assisted) between groups. Unpaired t test was used to find out the difference in quality of life domains and total score between groups. Microsoft Excel and Word were used to generate graph and tables.

\section{Results}

A total of 200 subjects were recruited for the study of which 135 were males and 65 were females. The mean age in group A was 63.69 and mean age in group B was 58.80 which was statistically significant $(\mathrm{p}<.001)$. Analysis of association of demographic variables between groups showed that gender, education, occupation, side, site and type of lesion did not influence the study (p>.05). Table I, Figure $1 \& 2$

Analysis of association of physical variables between groups showed that pain $\&$ independence in gait influenced the study $(\mathrm{p}<.05)$ and speech \& depression did not influence the study (Table II). Comparison of SSQOL domain between groups showed that group B had a better QOL life than group A which was statistically significant $(\mathrm{p}<.05)$ table III \& graph III. The total score of group A was 159.29 and total score in group B was 182.82 which was statistically significant $(\mathrm{p}<.0001)$. 
Table I: Baseline data for demographic variables

\begin{tabular}{|l|c|c|c|c|}
\hline Sl.No. & Variable & $\begin{array}{c}\text { Group A } \\
\text { (Without Early Rehab) }\end{array}$ & $\begin{array}{c}\text { Group B } \\
\text { (With Early Rehab) }\end{array}$ & p-value \\
\hline 1 & Number & 59 & 141 & - \\
\hline 2 & Age & $63.69 \pm 9.97$ & $58.80 \pm 9.26$ & $<0.001$ \\
\hline 3 & Gender(M/F) & $41 / 18$ & $95 / 46$ & $>0.770$ \\
\hline 4 & Side(Right/Left) & $38 / 21$ & $74 / 67$ & $>0.121$ \\
\hline 5 & Type(Ischemic/Hemorrhage) & $54 / 5$ & $123 / 18$ & $>0.386$ \\
\hline 6 & Site(MCA/ACA/PCA) & $53 / 5 / 1$ & $132 / 3 / 6$ & $>0.130$ \\
\hline
\end{tabular}

Graph I: level of education among both groups

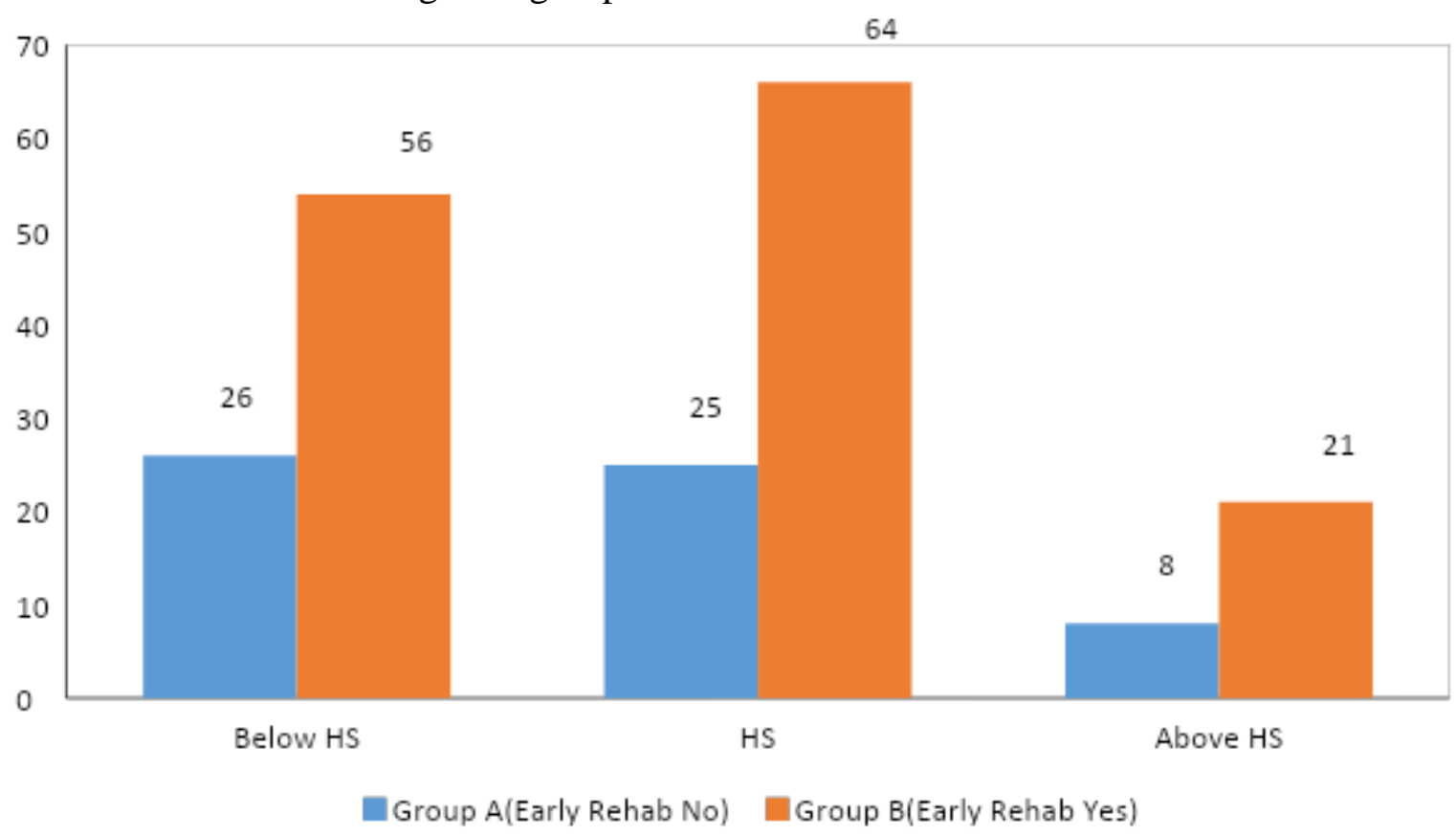

Graph 2: occupation among both groups

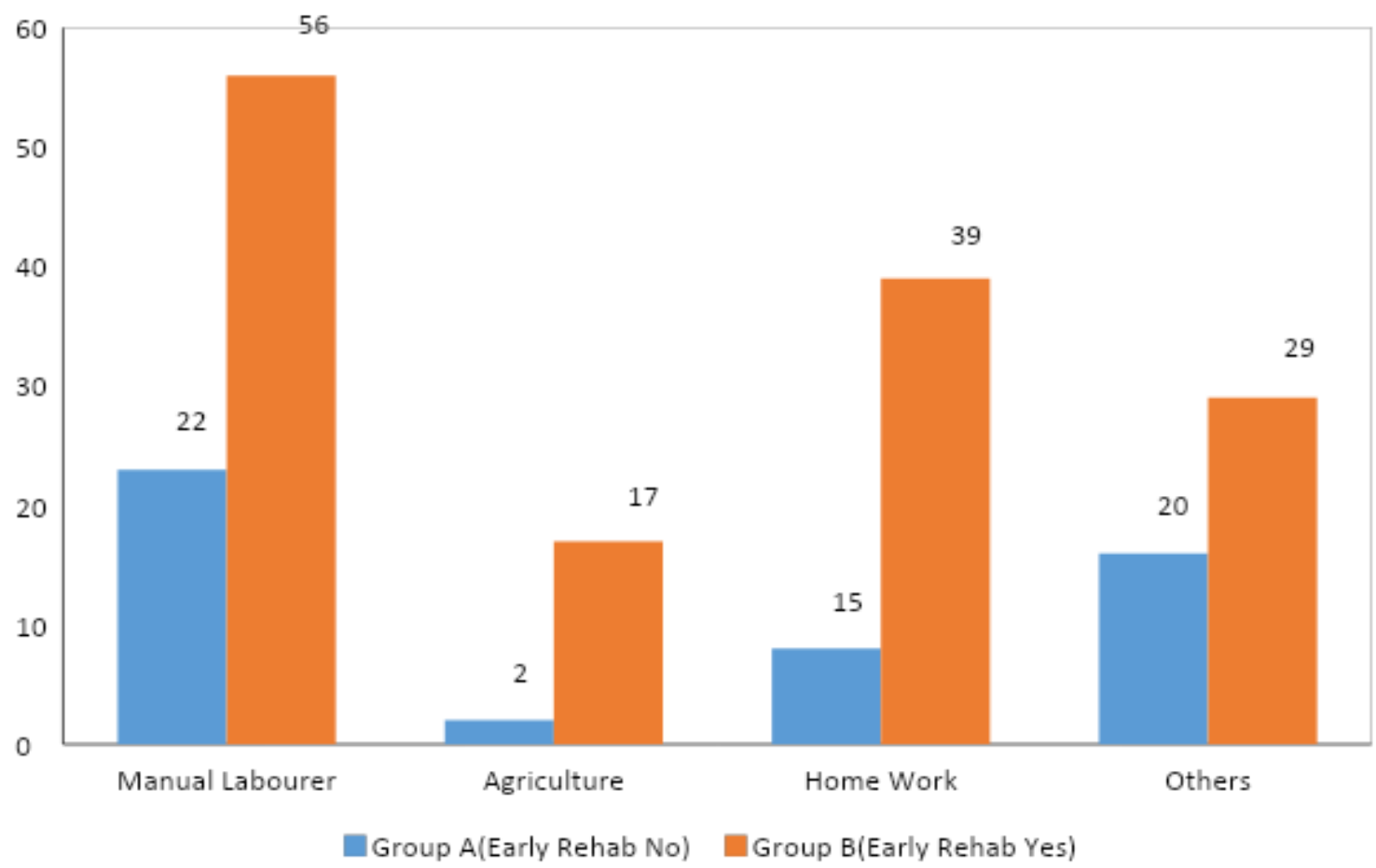


Table II: Physical variables among both groups

\begin{tabular}{|l|c|c|c|c|}
\hline Sl.No & Variable & Group A(Without Early Rehab) & Group B(With Early Rehab) & D-value \\
\hline 1 & Speech(No/Yes) & $34 / 25$ & $81 / 60$ & $>0.981$ \\
\hline 2 & Pain(No/Yes) & $51 / 8$ & $65 / 76$ & $<0.0001$ \\
\hline 3 & Depression(No/Yes) & $47 / 12$ & $104 / 37$ & $<0.376$ \\
\hline 4 & Gait(Independent /Assisted) & $39 / 20$ & $139 / 2$ & $>0.0001$ \\
\hline
\end{tabular}

Table III: Comparison of SSQOL domain between groups

\begin{tabular}{|l|c|c|c|c|}
\hline Sl.No: & $\begin{array}{c}\text { SSQOL } \\
\text { Domain }\end{array}$ & $\begin{array}{c}\text { Group A } \\
\text { (Without Early Rehab) }\end{array}$ & $\begin{array}{c}\text { Group B } \\
\text { (With Early Rehab) }\end{array}$ & P-value \\
\hline 1 & Energy & $9.03 \pm 2.03$ & $10.89 \pm 1.98$ & $<0.0001$ \\
\hline 2 & Family role & $9.76 \pm 2.25$ & $11.68 \pm 2.10$ & $<0.0001$ \\
\hline 3 & Language & $15.75 \pm 3.61$ & $17.52 \pm 3.94$ & $<0.003$ \\
\hline 4 & Mobility & $17.53 \pm 3.29$ & $20.43 \pm 3.99$ & $<0.0001$ \\
\hline 5 & Mood & $17.98 \pm 3.73$ & $21.01 \pm 3.40$ & $<0.0001$ \\
\hline 6 & Personality & $10.24 \pm 2.09$ & $12.09 \pm 1.89$ & $<0.0001$ \\
\hline 7 & Self-Care & $18.03 \pm 2.66$ & $19.97 \pm 2.85$ & $<0.0001$ \\
\hline 8 & Social role & $15.61 \pm 4.12$ & $17.74 \pm 3.71$ & $<0.0001$ \\
\hline 9 & Thinking & $10.49 \pm 1.87$ & $11.61 \pm 1.64$ & $<0.0001$ \\
\hline 10 & UE Function & $16.22 \pm 2.87$ & $17.80 \pm 3.24$ & $<0.001$ \\
\hline 10 & Vision & $11.71 \pm 2.00$ & $12.94 \pm 1.63$ & $<0.0001$ \\
\hline 11 & Work & $6.93 \pm 2.32$ & $9.13 \pm 2.69$ & $<0.0001$ \\
\hline
\end{tabular}

Graph 3: Mean total score of SSQOL among both groups

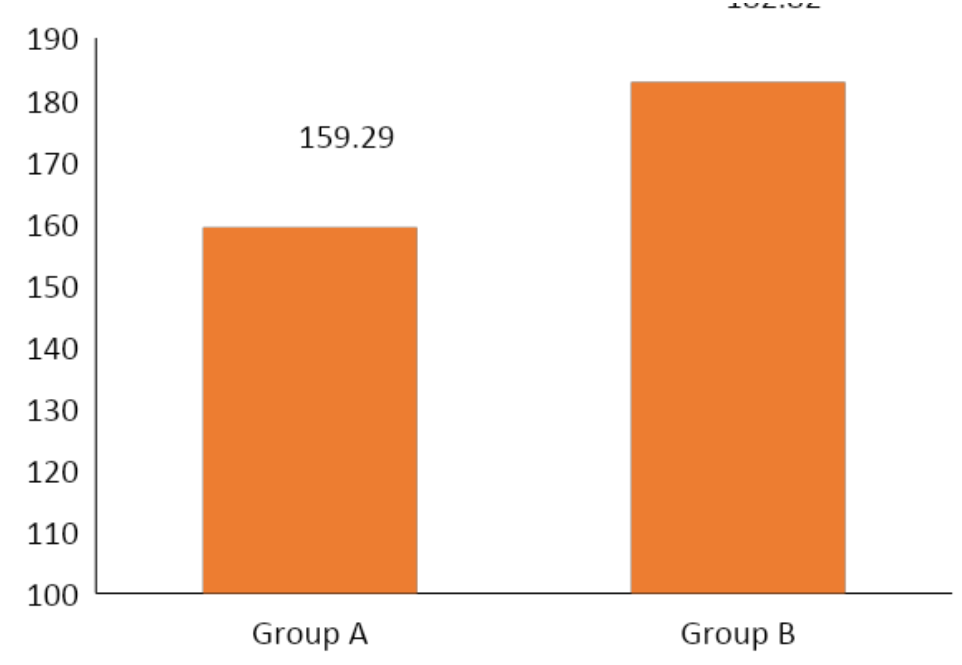

Total Score

\section{Discussion}

Of the 200 subjects in our study 141 had early rehabilitation. The mean age of patients in this group was 58.8 years and the mean age of those who did not receive early rehabilitation was 63.6 years. This suggests that there is inadequacy of early rehabilitation intervention in older patients in our population and this is statistically significant. The reasons for this difference has to be worked out. Dependency, architectural, economic and physical barriers, comorbidities etc. may be the responsible factors. Since the older people are more susceptible, appropriate measures to ensure early rehabilitation has to be ensured while planning treatment of stroke patients. 
Table IV: 2x2 table showing Association of early rehabilitation received or not with SSQOL

\begin{tabular}{|l|c|c|c|c|}
\hline \multirow{2}{*}{$\begin{array}{l}\text { Early rehabilitation } \\
\text { received }\end{array}$} & \multicolumn{2}{|c|}{ SSQOL } & \multirow{2}{*}{ Total } & \multirow{2}{*}{ p value } \\
\cline { 2 - 3 } & Low & Satisfactory & & \\
\hline No & 24 & 35 & 59 & \multirow{2}{*}{$<0.0001$} \\
\hline Yes & 11 & 130 & 141 & \\
\hline Total & 35 & 165 & 200 & \\
\hline
\end{tabular}

Association of early rehabilitation received or not with SSQOL was statistically significant $p$ value less than 0.0001. Those who received early rehabilitation achieved satisfactory quality of life. Out of 141 subjects, who received early rehabilitation 130 scored satisfactory quality of life and 11 scored low quality of life.

Quality of life was assessed in various domains using SSQOL questionnaire. Subjects with early rehabilitation showed better scores in all the domains. The quality of life in stroke survivors generally showed sub optimal levels with maximum decline in work and productivity, followed by mobility, social roles and language $e^{20}$. In analysis of our subjects without early rehabilitation, the most affected domain was again work and productivity. Energy, family roles and personality, mood, mobility and social roles are affected in that order. Those who underwent early rehabilitation had higher scores in all domains with a maximum difference in work and productivity. This has statistical significance. Early rehabilitation focussed on motor, speech, upper extremity functions etc. But the psychological and social factors were less attended. These results provided a reflection on the need for introduction of rehabilitation early during the course of treatment in stroke including psychosocial domains also.

The mean total score of different domains in the subjects without early rehabilitation was 159.29 (Group A) and those with early rehabilitation (Group B) was 182.82.The association between receiving early rehabilitation or not with SSQOL was statistically significant. $40.67 \%$ of subjects who did not receive early rehabilitation had low quality of life (score <147), while only $7.8 \%$ of subjects with early rehabilitation had low quality of life. In our study, rehabilitation initiated within the first week of stroke was considered as early rehabilitation. This is reported to improve mobility, decrease spasticity, prevent shoulder hand syndrome, minimizes contracture etc ${ }^{21}$. Interventions like positioning, early mobilisation, functional training, physical and occupational therapies, use of orthotics, interventions for speech etc. forms the basis in early rehabilitation interventions. Rehabilitation is a complex process providing quality care to the stroke survivor intending to improve functional status, level of independence and thereby quality of life. Rehabilitation is not an intervention to be introduced after every other modality is initiated in stroke care ${ }^{13}$. It is recommended to initiate rehabilitation as early as possible when the patient becomes medically stable and able to participate in active rehabilitation programs. Quality of life has been found to be better in patients who received early rehabilitation.

Males constitute the majority in both of our groups. $69.5 \%$ among those without early rehabilitation and $66.6 \%$ of subjects with early rehabilitation are males. Generally, women tend to have poorer outcomes. This is attributed to increased longevity, higher age of onset and socio- economic factors ${ }^{22}$. Social factors influencing quality of life in the stroke survivors are social isolation, living alone, inadequate caregiver support etc. However our study did not show any association for gender in early rehabilitation of stroke patients. Similarly, the other variables studied like side, site and type of lesion also did not significantly influence early rehabilitation. Though the side of stroke had influence on motor, sensory, speech and locomotion it was not reflected in our subjects to the extent of affecting the quality of life at this stage.

In the present study, four physical variables were evaluated for association with quality of life between the two groups A (Without early 
rehabilitation) and B (With early rehabilitation). Speech, pain, treatment for depression and independence in gait were the variables studied. Post stroke pain is disabling and reduces the quality of life ${ }^{23} .76$ of our subjects in Group B and 8 in Group A had pain. This was found to be statistically significant. Pain is a common association of stroke in the form of shoulder pain due to spasticity, subluxation, periarthritis etc., Central pain and Complex Regional Pain Syndrome. Early rehabilitation focussed on spasticity control, mobilisation and pain relief and was supervised. The level of pain perception might have been influenced by ongoing therapies and increased awareness about pain perception in Group B patients. Pain could also be attributed to other causes like osteoarthritis, trauma, mechanical, psychogenic etc., the details of which were not elicited in our data collection. These might have led to the increased number of subjects with pain in Group B. this reflects on the necessity of focussed pain management as a part of stroke rehabilitation programme. $11 \%$ of our subjects had assisted gait and variable levels of dependency for locomotion. The influence of early rehabilitation on gait was significant. Only $1.4 \%$ of subjects who received early rehabilitation had assisted gait while $33 \%$ of those without early rehabilitation had assisted gait. All our patients had motor impairment which significantly improved with time and the recovery was enhanced with early rehabilitation intervention. The independence in gait is influenced by spasticity, range of joint mobility, weakness, awareness and motivation of the patient and care giver. During the phase of early rehabilitation these issues were addressed effectively and this provided more independence in gait in a majority of the subjects who received early rehabilitation.

Speech is the most common form of communication and defective comprehension, expression and articulation negatively influences a person's functional independence. Our study did not show a significant influence of early rehabilitation on speech. Lack of proper speech therapy, impaired cognition, literacy level etc. could account for this. Depression in the early phase of stroke retards recovery and negatively influences rehabilitation.24.5\% of our subjects had received antidepressant treatment while in the course of the treatment for stroke.

There are limitations for this study. The subjects were selected directly from a rehabilitation unit and hence might be under sampled. There was no consideration for rural urban variations. Socio economic factors which restrain rehabilitation treatment were also not considered. Grading of severity of the lesion was not taken into account during data collection. All patients with global aphasia were excluded. Moreover the exact nature of early rehabilitation was not elucidated.

Further follow up studies may be undertaken at 6 , 9 and 12 months to assess the functional level and quality of life of the present subjects. Studies can be done to compare the quality of lives in stroke subjects who underwent supervised and nonsupervised rehabilitation programs.

\section{Conclusion}

The study assessed the difference in quality of life in stroke subjects who received early rehabilitation and those who did not. The total SSQOL score was more in subjects who received early rehabilitation indicating their better quality of life compared to the other group. The scores were better in all the domains in this group. The mean age in the group which received early rehabilitation was less, which was statistically significant. All other demographic variables were not associated with early rehabilitation. Of the physical variables, pain and independence in gait showed significant association with early rehabilitation among both groups of stroke survivors. Those who received early rehabilitation were more independent in gait compared to others. Those who received early rehabilitation had a better perception of pain compared to others. This highlights the role of early rehabilitation in stroke care and the necessity of a complete stroke care pathway with initiation of 
rehabilitation as soon as the patient becomes medically fit to undergo active rehabilitation programs.

\section{References}

1. King R.B. Quality of life after stroke. Stroke.1996 Sep;27(9):1467-72

2. Stroke Statistics|Internet Stroke Center [Internet]. cited 2018 Jun20, 10 AM] Available

from:http://www.strokecenter.org/patients/ about-stroke-statistics.

3. Vasantha M Padma, Rohit Bhatia.et al. call for neurologists to take up stroke interventions. Ann Indian Acad Neurol.2016 Oct- Dec;19(4):429-4328

4. Feigin VL et.al. Worldwide stroke incidence and early case fatality reported in 56 population-based studies: A systemic review. Lancet Neurol.2009;8:355-69

5. Fergin, Bo Norrving, George A Mensa. Global burden of stroke. Circulation Research, February 3, 2017;439

6. Eitan Auriel,Natan M Bornstein. Early Mobilisation Following stroke. European Neurological review 2013;8(2):141-3

7. Hebert $D$ et.al. Canadian stroke best practice recommendations: Stroke rehabilitation practice guidelines, update 2015. International Journal of Stroke. Vol.11(4)459-484

8. Mohammed Alim.et.al. Family-led rehabilitation after stroke in India: the ATTEND trial, study protocol for a randomized controlled trial.Trials.2016; $17: 13$

9. Siew Kwaon Lui and Minh Ha Nguyen. Elderly Stroke Rehabilitation: Overcoming the Complications and Its Associated Challenges. Curr Gerontol Geriatr Res. 2018; 2018: 9853837.

10. Elisheva R. Coleman.et.al. Early Rehabilitation After Stroke: a Narrative Review. Curr Atheroscler Rep. 2017 Nov 7; 19(12): 59.
11. Cathy Stinear, Suzanne Ackerley and Winston Byblow. Rehabilitation is Initiated Early After Stroke, but Most Motor Rehabilitation Trials Are Not. A Systematic Review. Stroke. 2013; 44: 2039-2045

12. Sundseth A, Thommessen B, Ronning OM. Outcome after mobilization within 24 hours of acute stroke: a randomized controlled trial. Stroke. 2012；43：23892394.

13. Hayes SH, Carroll SR: Early intervention care in acute stroke patient. Arch Phys Med Rehabil67:319- 321

14. WHO QOL group, Development of the World Health Organization WHOQOLBREF quality of life assessment. Psychol Med. 1998: 28: 551-558.

15. Moon, Kim.et.al. Correlates of quality of life after stroke. Journal of the Neurological Sciences.2004; 224:37-41

16. Kamel A, Ghani AA, Zaiton MA, ElMotayam AS, El-Fattah DA. Health related quality of life in stroke survivors measured by the Stroke Impact Scale. Egypt J Neurol Psychiatry Neurosurg. 2010; 47:267-74.

17. JA Opara and K Jaracz. Quality of life of post-stroke patients and their caregivers. J Med Life.2010 Aug15;3(3):216-220 [Published online2010 Aug 25]

18. 18. William $G$ Akanksha et al. Clinical Features, Risk Factors, and Short- term outcome of Ischemic Stroke in Patients with Atrial Fibrillation: Data from a Population- based Study. Ann Indian Acad Neurol.2017 July-Sep;20(3):289-293

19. Edja Solange Souza Rangel; Angélica Gonçalves Silva Belasco; Solange Diccini. Quality of life of patients with stroke rehabilitation.

Available from: http://www.scielo.br/scielo.php?pid=S010 3-21002013000200 016\& script $=$ sci_arttext\&tlng=en 
20. Dr PC Muralidharan et.al. Quality of Life in Stroke Survivors in Central Kerala. JMSCR Volume 07 Issue 06 June 2019 986-995

21. Pan, Xiao-Li, MD. Efficacy of early rehabilitation therapy on movement ability of hemiplegic lower extremity in patients with acute cerebrovascular accident. Medicine: January 2018 Volume 97 - Issue 2 - p e9544

22. MJ Reeves, et.al. Sex differences in stroke: epidemiology, clinical presentation, medical care, and outcomes. Lancet Neurol.2008 Oct;7(10):915-926

23. Aprile et.al. Pain in stroke patients: characteristics and impact on the rehabilitation treatment. A multicentral cross-sectional study. Eur J Phys Rehabil Med 2015 Feb 26 51(6),725-36. 